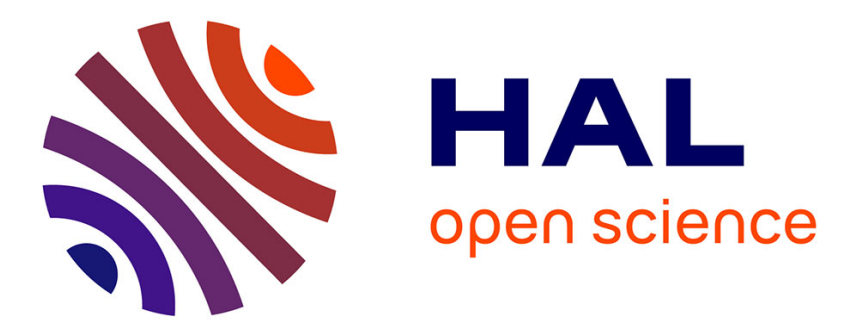

\title{
Evolution de la granulométrie des dépôts discontinus d'or sur substrats de $\mathrm{MgO}$
}

\author{
A. Carlan, G. Desrousseaux, B. Vidal, A. Renou
}

\section{To cite this version:}

A. Carlan, G. Desrousseaux, B. Vidal, A. Renou. Evolution de la granulométrie des dépôts discontinus d'or sur substrats de MgO. Revue de Physique Appliquée, 1989, 24 (7), pp.693-698. 10.1051/rphysap:01989002407069300 . jpa-00246093

\section{HAL Id: jpa-00246093 https://hal.science/jpa-00246093}

Submitted on 1 Jan 1989

HAL is a multi-disciplinary open access archive for the deposit and dissemination of scientific research documents, whether they are published or not. The documents may come from teaching and research institutions in France or abroad, or from public or private research centers.
L'archive ouverte pluridisciplinaire HAL, est destinée au dépôt et à la diffusion de documents scientifiques de niveau recherche, publiés ou non, émanant des établissements d'enseignement et de recherche français ou étrangers, des laboratoires publics ou privés. 


\title{
REVUE DE PHYSIQUE APPLIQUEEE
}

Revue Phys. Appl. 24 (1989) 693-698

JUILLET 1989, PAGE 693

Classification

Physics Abstracts

$73.40 \mathrm{G}-73.60 \mathrm{D}-73.90$

\section{Evolution de la granulométrie des dépôts discontinus d'or sur substrats de MgO}

\author{
A. Carlan ( $\left.{ }^{1}\right)$, G. Desrousseaux ( $\left.{ }^{2}\right)$, B. Vidal ( $\left.{ }^{1}\right)$ et A. Renou ( $\left.{ }^{2}\right)$ \\ (1) Laboratoire des Propriétés Physiques des Couches Minces, Université d'Aix-Marseille III, Faculté des \\ Sciences et Techniques de Saint-Jérome, Avenue Escadrille Normandie Niemen, 13397 Marseille Cedex 13, \\ France \\ ${ }^{(2)}$ Laboratoire de Microscopie et Diffractions Electroniques, Université d'Aix-Marseille III, Faculté des \\ Sciences et Techniques de Saint-Jérome, Avenue Escadrille Normandie Niemen, 13397 Marseille Cedex 13, \\ France
}

(Reçu le 21 novembre 1988, révisé le 3 mars 1989, accepté le 29 mars 1989)

\begin{abstract}
Résumé. - Dans nos précédents travaux la géométrie instantanée que présente sous flux les condensats métalliques était déterminée par itération à partir des mesures conjointes de la conductance de couche $C_{\mathrm{c}}$ et de l'épaisseur massique $e_{\mathrm{D}}$. A présent, nous nous proposons d'obtenir une vue d'ensemble de l'évolution géométrique en superposant à des abaques les courbes $C_{\mathrm{c}}\left(e_{\mathrm{D}}\right)$ relatives à divers systèmes $\mathrm{Au} / \mathrm{MgO}$. Ce procédé permet de mettre rapidement en évidence les nucléations primaires et secondaires et à plus haute température de condensation la croissance homothétique des agrégats.
\end{abstract}

\begin{abstract}
In our previous works the instantaneous geometry which the metal condensates show under atomic flux is computed by iteration from both the sheet conductance and the mass thickness $e_{\mathrm{D}}$. Now our purpose is to get a general view over geometrical evolution by surimposing over abacus of curves $C_{\mathrm{c}}\left(e_{\mathrm{D}}\right)$ relative to several $\mathrm{Au} / \mathrm{MgO}$ systems. This procedure enables us to quickly emphasize the primary and secondary nucleations and the homothetic growth of aggregates at higher condensation temperatures.
\end{abstract}

\section{Introduction.}

Le processus de formation des dépôts métalliques sur différents supports varie principalement d'une part avec la nature du condensat et de son substrat d'autre part avec le mode de préparation et de ses conditions (flux, température du substrat...).

Durant les vingt dernières années, la croissance des couches minces a été très étudiée soit in situ en cours de formation, soit de façon séquentielle par prélèvement d'échantillons. Les grandeurs granulométriques qui permettent de décrire quantitativement la répartition du métal déposé, ont été déterminées de différentes manières. La microscopie électronique par transmission permet de révéler la forme des agrégats et les distributions des tailles et des distances [1,2]. La conduction électrique [3] et l'absorption optique $[4,5]$ donnent une vision plus globale de la géométrie du condensat.

Dans le présent travail, nous étudions à partir de mesures électriques les processus de formation et de croissance des dépôts métallique d'or sur support de $\mathrm{MgO}$. La méthode électrique utilisée a déjà été décrite [6]. Elle permet de suivre en continu les variations de la conductance de couche $C_{\mathrm{c}}$ et de l'épaisseur du dépôt $e_{\mathrm{D}}$. Le calcul montre que le courant d'effet tunnel à travers le substrat est nettement supérieur à celui que pourrait permettre une émission thermoionique [7]. Le principal processus de conduction est donc l'effet tunnel à travers le substrat de $\mathrm{MgO}$. Nous pouvons alors connaître l'état granulométrique du dépôt d'or sur $\mathrm{MgO}$ à chaque instant à partir des mesures de conductance de couche et d'épaisseur massique par application des relations précédemment publiées [6]. Utilisant l'expression de l'intensité du courant en fonction de l'épaisseur du dépôt, on constitue un abaque (Fig. 1) représentant les variations $I=f\left(e_{\mathrm{D}}\right)$ paramétrées par :

- soit la densité d'agrégats $n_{\mathrm{g}}$;

- soit le diamètre moyen $a_{\|}$;

- soit enfin la distance bord à bord $s$. 


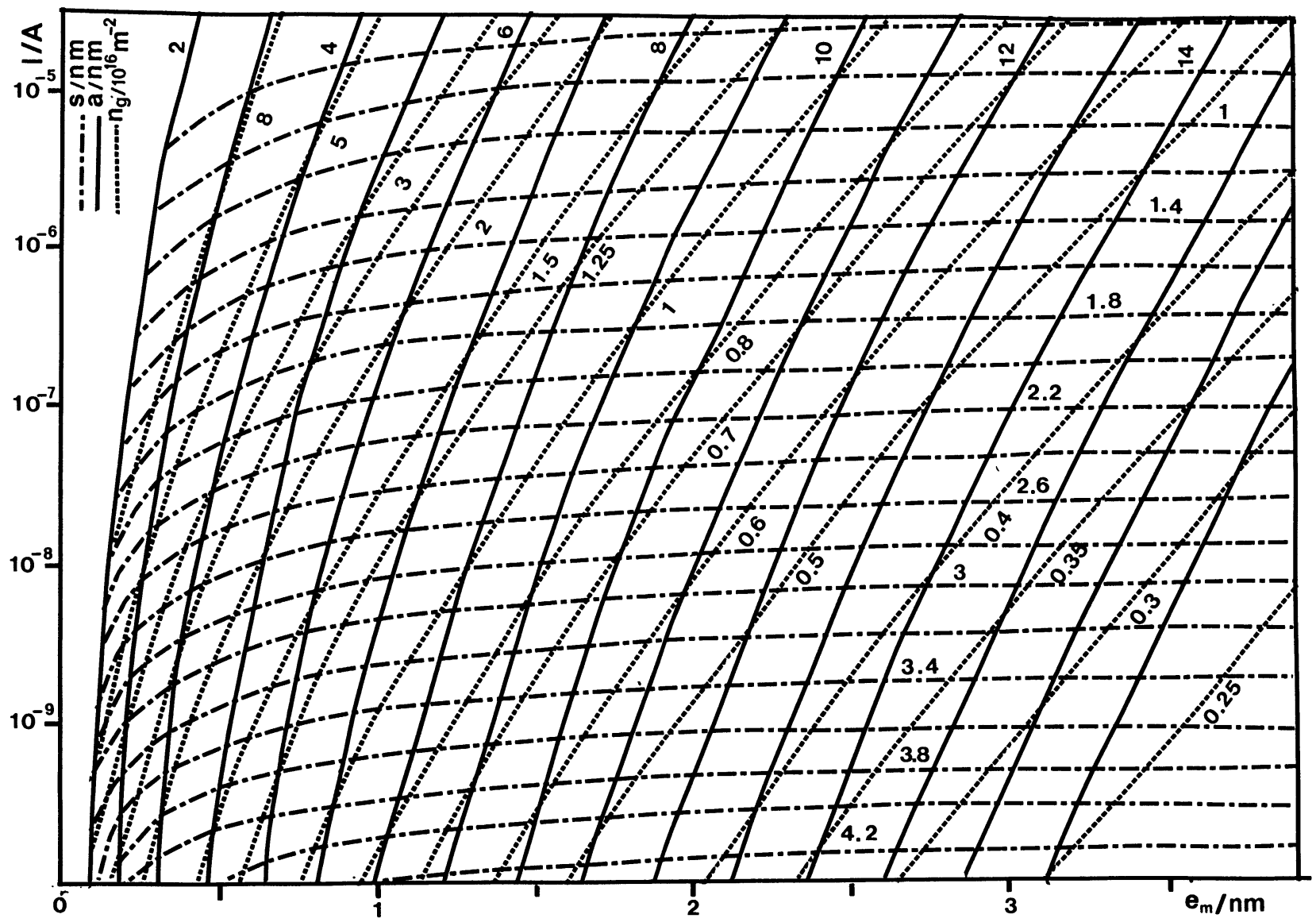

Fig. 1. - L'intensité du courant d'effet tunnel $I$ traversant le dépôt métallique granulaire dans son plan est représentée en fonction de $e_{\mathrm{D}}$ pour des valeurs constantes soit de $s$, soit de $a_{\mathrm{l}}$, soit de $n_{\mathrm{g}} \cdot\left(I=5 \Delta C_{\mathrm{c}}\right)$.

[The tunnel current intensity flowing in the plane of the granular metal condensate is plotted versus $e_{\mathrm{D}}$ at constant values respectively of $s, a_{1}$, and $n_{\mathrm{g}} \cdot\left(I=5 \Delta C_{\mathrm{c}}\right)$.]

La confrontation des résultats expérimentaux aux abaques ainsi construits donne directement une vue d'ensemble sur les processus de croissance des dépôts d'or sur $\mathrm{MgO}$. Lors de la capture des atomes incidents et des adatomes, les variations $I=f\left(e_{\mathrm{D}}\right)$ peuvent se produire à $a_{\|}=$constante, $n_{\mathrm{g}}=$ constante ou $s=$ constante.

Lorsque $a_{\|}$varie peu la courbe expérimentale présente l'allure des traits pleins de l'abaque : il y a nucléation primaire. Lorsque $s$ varie peu, la croissance du diamètre $a_{\|}$des grains immobiles «croissance statique " n'a pour effet que de réduire le nombre d'espaces intergranulaires et par conséquent la densité d'agrégats $\boldsymbol{n}_{\mathrm{g}}$.

Enfin, si la population des grains se stabilise $\left(n_{\mathrm{g}}=\right.$ constante $)$, le diamètre $a_{\|}$ne pourra croître qu'aux dépens de $s$ (coalescence statique). La variation de $I$ avec $e_{\mathrm{D}}$ adoptera l'allure des lignes tiretées de l'abaque. Ces processus peuvent se combiner ou alterner au cours d'une même condensation.

\section{Rappels théoriques du modèle granulométrique des dépôts métalliques.}

La description quantitative de la granulométrie du condensat métallique durant sa formation sous vide impose une modélisation de la forme des grains et de leur répartition sur le substrat. Les grains sont assimilés à des ellipsoïdes tronqués présentant une symétrie de révolution autour d'un axe normal au substrat. Ils sont approximativement centrés aux nœuds d'un réseau triangulaire.

Dans une telle configuration la distance centre à centre a pour expression :

$$
\ell=a_{\|}+s=2 \sqrt{3} n_{\mathrm{g}}^{-1 / 2} .
$$

Le volume de métal déposé par unité d'aire du substrat ou épaisseur massique $e_{\mathrm{D}}$ s'exprime alors par la relation: $e_{\mathrm{D}}=v_{0} q t=g n_{\mathrm{g}} a^{3}$ où $g$ est le facteur de forme du grain et $v_{0}$ le volume atomique. 
Ce facteur est fonction de l'excentricité et de l'angle de troncature du grain [7]. La vitesse de dépôt du métal est la quantité $q$ d'atomes d'or déposés par seconde sur l'unité d'aire du substrat.

Nous avons ensuite exprimé en fonction des grandeurs granulométriques la variation $C_{\mathrm{c}}$ de la conductance de couche du système dépôt-substrat :

$$
C_{\mathrm{c}}=\left(D_{\mathrm{E}} / L_{\mathrm{E}}\right) \ell / U_{\mathrm{E}}
$$

$U_{\mathrm{E}}$ est la tension constante entre deux électrodes présentant perpendiculairement au champ moyen la dimension $L_{\mathrm{E}}=2 \mathrm{~cm}$ et entre elles une distance $D_{\mathrm{E}}=2 \mathrm{~mm}$.

$I$ représente la variation de l'intensité du courant due à la présence du dépôt métallique.

Pour interpréter les résultats expérimentaux, il semble intéressant de représenter les variations de $I$ en fonction de l'épaisseur $e_{\mathrm{D}}$ ou du nombre d'atomes déposés $n_{\mathrm{a}}$ de manière à pouvoir les comparer aux abaques de la figure 1 .

Il est à signaler d'une part que la taille des amas métalliques susceptibles d'être pris en compte dans l'effet tunnel via le substrat est suffisante pour ne pas avoir à considérer les effets de localisation. D'autre part les phénomènes de capillarité maintiennent une géométrie du condensat loin du seuil de percolation pour les épaisseurs massiques utilisées. C'est ce que l'on a pu vérifier à l'examen des clichés de Microscopie Electronique.

Dans le cas de nos dépôts d'or sur $\mathrm{MgO}$, nous avons retenu une hauteur moyenne de barrière de $0,83 \mathrm{eV}$, une masse effective de l'électron franchissant cette barrière $m=0,1 m_{0}$ et une permittivité du substrat $\varepsilon=9,8 \varepsilon_{0}$. Ces valeurs extraites de différentes publications [8-10] nous ont paru acceptables dans le cadre de nos expériences.

\section{Résultats expérimentaux.}

La préparation des substrats de $\mathrm{MgO}$ est réalisée au canon à électrons dans une enceinte à ultravide. L'or est déposé sur $\mathrm{MgO}$ à une vitesse de $0,4 \AA / \mathrm{min}$ à l'aide d'une cellule de Knudsen préalablement étalonnée à $0,01 \AA ̊ / \mathrm{min}$ près.

L'ensemble des opérations est réalisée sans rupture de vide après avoir atteint une pression de $10^{-6} \mathrm{~Pa}$ en début de chaque évaporation. Pour chacune des préparations les conditions de condensation sont données dans le tableau I.

Nous présentons en figure 2 les résultats de neuf expériences dont nous examinerons l'évolution granulométrique. Les figures $3 a$ et $3 b$ montrent les clichés de Microscopie Electronique des préparations 5 et 8.

La confrontation des variations de l'intensité du courant en fonction de l'épaisseur massique aux abaques de la figure 1 fait apparaître différentes phases dans le processus de formation des couches.

Les courbes 1,2 et 7 sont sensiblement parallèles à celles de la famille, tracées à diamètre $a_{\|}$constant. Par ailleurs leurs pentes surpassant celles tracées à densité $n_{\mathrm{g}}$ constante montrent que la densité d'agrégats croît avec l'épaisseur $e_{\mathrm{D}}$. Ceci suggère d'ores et déjà un processus de nucléation primaire.

Pour les dépôts notés $4,5,6$ et 8 on observe la diminution du diamètre moyen des agrégats pendant que leur concentration croît. Ceci est caractéristique de la nucléation secondaire après libération des sites. Les courbes 3 et 9 sont relatives à des dépôts d'or effectués sur des supports de $\mathrm{MgO}$ chauffés à $340 \mathrm{~K}$ environ. On rencontre à présent le cas de variations qui se produisent avec une densité d'agrégats $n_{\mathrm{g}}$ sensiblement constante tandis que le diamètre moyen des grains croît. Il y a grossissement sur place des agrégats.

Table I. - Conditions de condensation des neuf dépôts d'or sur $\mathrm{MgO}$.

[Conditions of condensation of the nine gold deposits on $\mathrm{MgO}$ substrates.]

\begin{tabular}{|c|c|c|c|c|c|c|c|c|c|}
\hline & 1 & 2 & 3 & 4 & 5 & 6 & 7 & 8 & 9 \\
\hline$q\left\{\begin{array}{c}\AA \mathrm{s}^{-1} \\
\mathrm{~cm}^{-2} \mathrm{~s}^{-1}\end{array}\right.$ & 0,0031 & 0,0053 & 0,00534 & 0,0079 & 0,016 & 0,045 & 0,00535 & 0,0096 & 0,0056 \\
\cline { 2 - 11 } & $1,83 \times 10^{12}$ & $3,11 \times 10^{12}$ & $3,15 \times 10^{12}$ & $4,66 \times 10^{12}$ & $9,44 \times 10^{12}$ & $26,55 \times 10^{12}$ & $3,16 \times 10^{12}$ & $5,66 \times 10^{12}$ & $3,30 \times 10^{12}$ \\
\hline$T_{\text {initiale }}$ & $305 \mathrm{~K}$ & $305 \mathrm{~K}$ & $340 \mathrm{~K}$ & $305 \mathrm{~K}$ & $305 \mathrm{~K}$ & $305 \mathrm{~K}$ & $305 \mathrm{~K}$ & $305 \mathrm{~K}$ & $340 \mathrm{~K}$ \\
\hline$T_{\text {finale }}$ & $312 \mathrm{~K}$ & $319 \mathrm{~K}$ & $340 \mathrm{~K}$ & $315 \mathrm{~K}$ & $310 \mathrm{~K}$ & $308 \mathrm{~K}$ & $326 \mathrm{~K}$ & $330 \mathrm{~K}$ & $340 \mathrm{~K}$ \\
\hline$P_{\text {initiale }}^{(\mu \mathrm{P})}$ & 1 & 1 & 1,33 & 1,0 & 1,0 & 1,33 & 1,33 & 1,33 & 1,33 \\
\hline $\begin{array}{c}P \text { finale } \\
(\mu \mathrm{P})\end{array}$ & 2 & 2 & 5 & 7 & 8 & 9 & 10 & 14 & 16 \\
\hline
\end{tabular}

Les dépôts 3 et 9 ont été faits sur supports chauffés. 


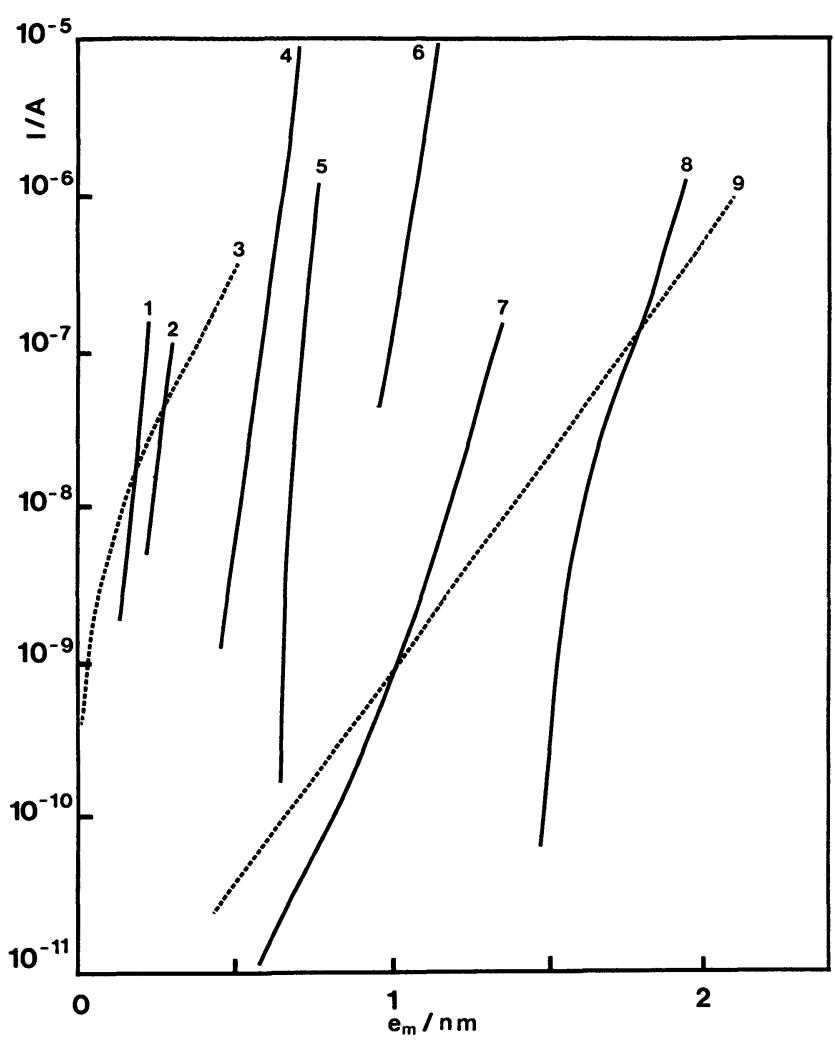

Fig. 2. - Variations du courant d'effet tunnel entre grains en fonction de l'épaisseur massique de neuf condensats d'or sur substrat de $\mathrm{MgO}$. Les courbes en pointillé signalent un chauffage du substrat à $340 \mathrm{~K}$.

[Variations of the planar tunnel current intensity flowing in nine granular metal condensates on $\mathrm{MgO}$ substrate is plotted versus their mass thickness $e_{\mathrm{D}}$. The dashed curves point out a heating of substrate at $340 \mathrm{~K}$.]

\section{Interprétation des résultats.}

Pour les dépôts sur isolant, le courant électrique d'effet tunnel reste très faible tant que la distance intergrain demeure très grande par rapport à leur diamètre moyen $a_{\|}$. C'est précisément le cas dans la première phase de formation du dépôt. Cette situation est analogue à celle de l'évolution de la conductance des cermets [11].

Lorsque la distance bord à bord $s$ décroît jusqu'à devenir comparable au diamètre d'agrégats, le courant électrique se met à croître fortement. ( $s$ est alors de l'ordre de quelques nanomètres). Si on compare aux abaques précédents les variations $I\left(e_{\mathrm{D}}\right)$ représentées par les courbes $1,2,4,5,6,7$ et 8 on constate que la densité d'agrégats crôit avec l'épaisseur massique $e_{\mathrm{D}}$. Il y a nucléation de l'or sur le support de $\mathrm{MgO}$.

Les courbes 1,2 et 7 montrent que le diamètre moyen $a_{\|}$varie peu pendant l'augmentation de la densité d'agrégats $n_{\mathrm{g}}$. Il y a nucléation primaire. En effet, la croissance de $n_{\mathrm{g}}$ entraîne la décroissance de la distance $a_{\|}+s$ et corrélativement pour un diamètre moyen sensiblement constant, celle de la distance intergrain $s$. Par contre, pour les dépôts notés 4, 5, 6 et 8 , le diamètre moyen $a_{\|}$décroît, ce qui suggère une nucléation secondaire sur sites libérés si $n_{\mathrm{g}}$ croît.

Dans le cas de la nucléation secondaire deux processus peuvent exister séparément ou simultanément. D'une part, lors de la coalescence dynamique l'excentricité des grains décroît avec leur grossissement, ce qui conduit à une diminution du diamètre moyen et une décroissance de $n_{\mathrm{g}}$. D'autre part, de nouveaux agrégats nucléent sur les sites libérés, ce qui a pour effet d'accroître la densité $n_{\mathrm{g}}$ et de diminuer le diamètre moyen. La croissance de $n_{\mathrm{g}}$ ne peut être assurée que par le second processus alors que les deux contribuent à diminuer le diamètre moyen.

Les croissances de l'intensité du courant avec l'épaisseur dans les relevés 3 et 9 montrent que la densité $n_{\mathrm{g}}$ varie peu avec l'épaisseur $e_{\mathrm{D}}$ tandis que le diamètre moyen croît. Il y a grossissement sur place des agrégats. En effet, l'abaque correspondant à ces variations présente pour une densité $n_{\mathrm{g}}$ constante une décroissance rapide de $s$ et une croissance du diamètre moyen $a_{\|}$.

\section{Conclusion.}

Les résultats obtenus lors du suivi de l'évolution granulométrique des dépôts d'or sur $\mathrm{MgO}$ ont montré que le processus de croissance de ces dépôts se présentait sous trois formes différentes.

Pour les préparations 1 et 2 le diamètre moyen des agrégats varie peu avec l'épaisseur lorsque leur densité $n_{\mathrm{g}}$ croît faiblement. La nucléation primaire reste prédominante. Pour les préparations $4,5,6,7$ et 8 le diamètre moyen diminue, la densité d'agrégats croît durant l'augmentation du nombre moyen d'atomes $n_{\mathrm{a}}$ par unité de surface. Il y a formation de nouveaux agrégats par occupation de sites actifs libérés par coalescence dynamique préalable. La nucléation secondaire a débuté.

Enfin la croissance des couches d'or 3 et 9 sur support de $\mathrm{MgO}$ chauffé s'est faite à densité de grains constante, c'est-à-dire par croissance homothétique des agrégats.

Les résultats que nous avons présentés montrent que, d'une préparation à une autre, le processus de formation des dépôts d'or sur $\mathrm{MgO}$ varie amplement avec les conditions de condensation. Les courbes 1 , 2 et 3 de la figure 2 correspondant à une faible couverture atomique $n_{\mathrm{a}}$ ne peuvent montrer qu'une nucléation primaire. Par contre, dans la partie droite de la figure 2 les phénomènes de coalescence ont pris une part importante et la nucléation secondaire a apparu.

Ainsi, cette méthode permet de rendre compte directement d'un large éventail de processus d'évolution de dépôts métalliques granulaires en cours de formation. 


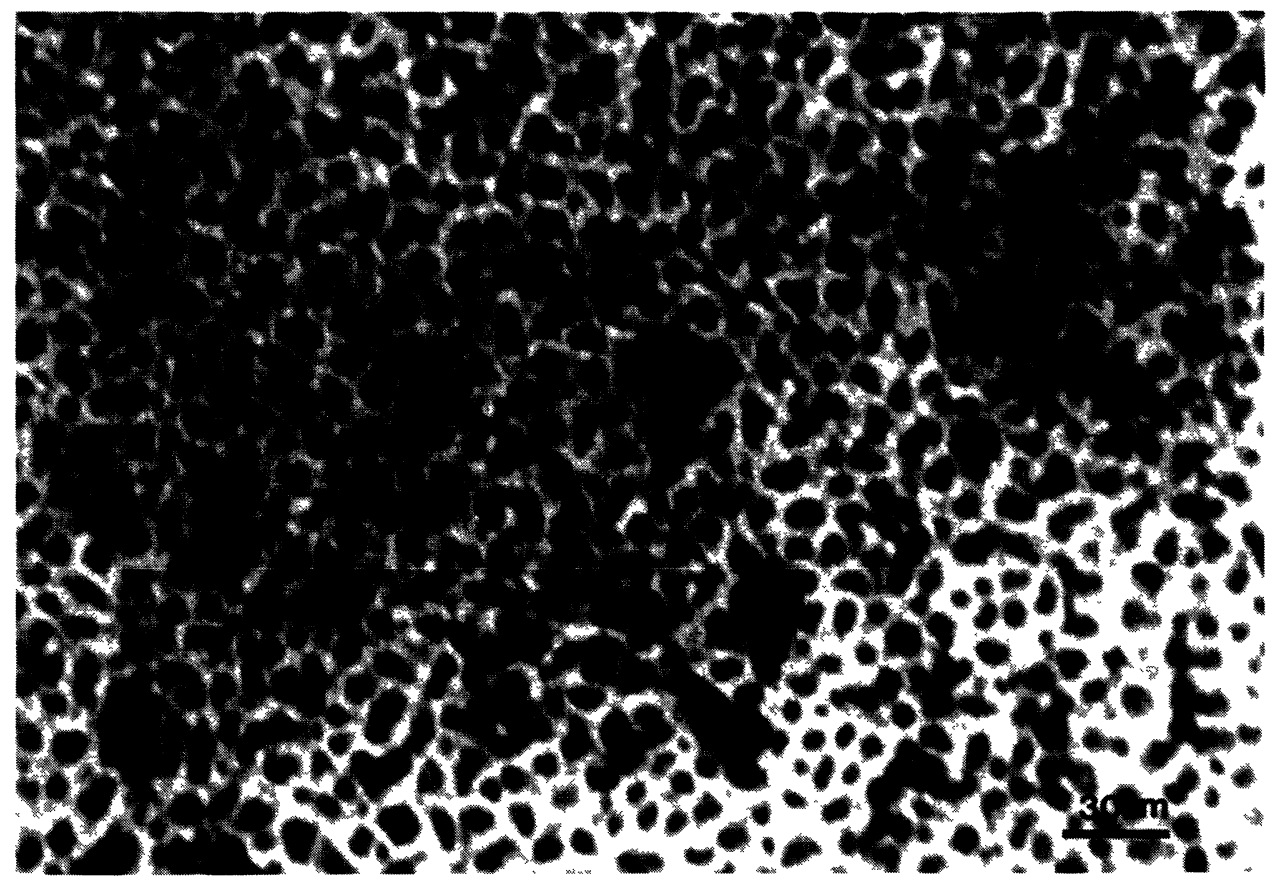

a)

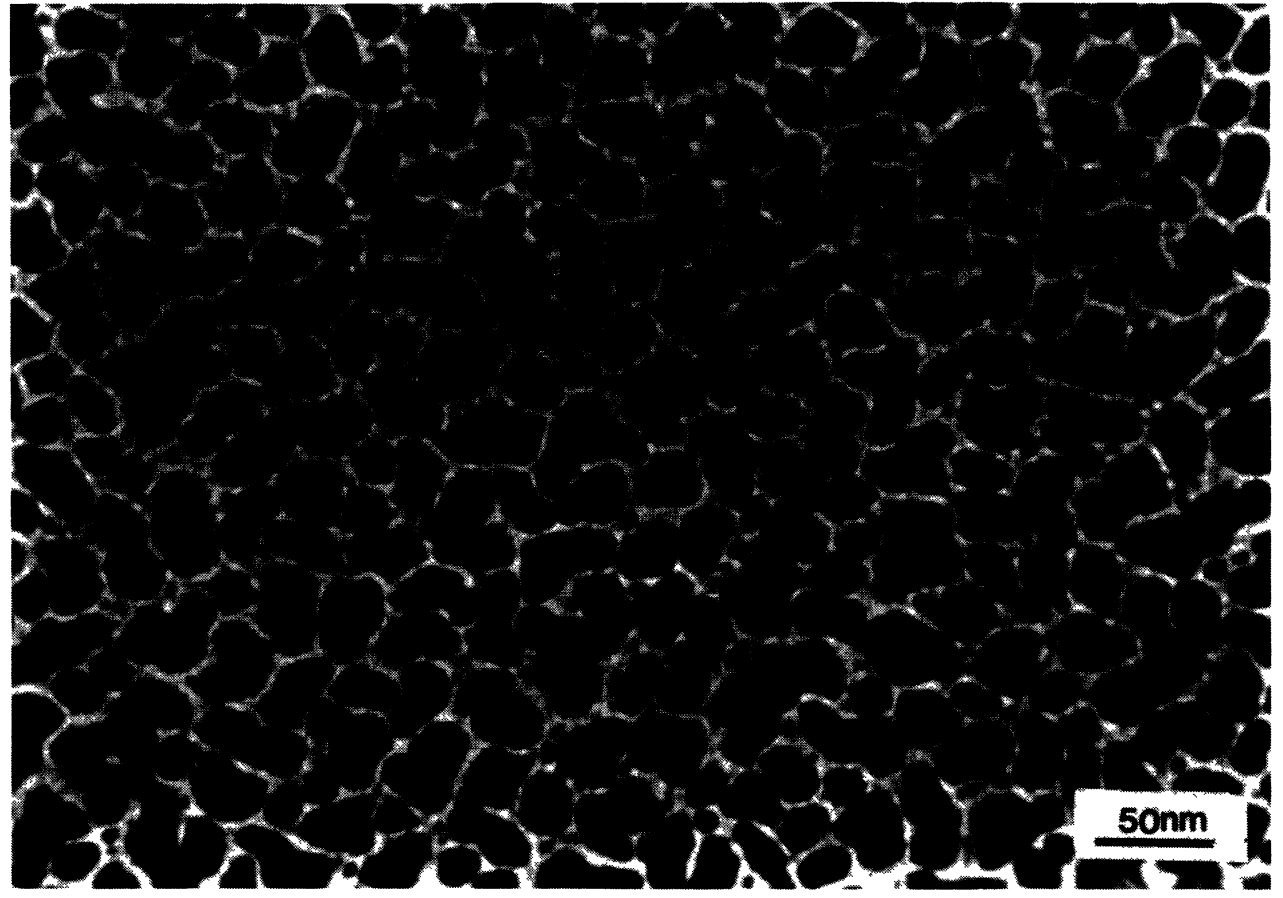

b)

Fig. 3. - (a) Cliché de Microscopie Electronique de la préparation No. 5. (b) Cliché de Microscopie Electronique de la préparation No. 8.

[(a) Electron Micrograph of $\mathrm{Au}$ deposit on $\mathrm{MgO}$ substrate No. 5. (b) Electron micrograph of $\mathrm{Au}$ deposit on $\mathrm{Mg}$ substrate No. 8.] 


\section{Bibliographie}

[1] Granquist C. G., Buhrman, R. A., J. Appl. Phys. 5 (1976) 2200.

[2] Anderson T., Granquist C. G., J. Appl. Phys. 4 (1977) 1673.

[3] Kazmerski L. L., Racine D. M., J. Appl. Phys. 46 (1975) 791.

[4] Norman S., Anderson T., Granquist C. G., Hunderi O., Solid State Commun. 23 (1977) 261.

[5] BARR A., FIney R. D., Thin Solid Films 24 (1974) S 11.

[6] Desrousseaux G., Carlan A., Robrieux B., Schaffar H., TrompetTe J., Dussaulcy J. P., Faure R., J. Phys. Chem. Solids 46 (1985) 929.
[7] Desrousseaux G., Trompette J., Faure R., Schaffar H., Dussaulcy J.P., Thin Solid Films 98 (1982) 139.

[8] Tirodkar P. P., Pratap R., Indian J. Pure Appl. Phys. 16 (1978) 582.

[9] Duke C. B., Solid State Phys. Suppl. 10 (1969); Tunneling in Solids, A.P.IV. 6, pp. 36-49.

[10] Mott N. F., GuRney R. W., Electronic Processes in Ionic Crystal, 2nd Ed. (Oxford University Press, Fair Lawn, N.J.) 1948, Chap. V.

[11] Abeles B., Ping Sheng, Coutts M. D., Arie Y., Adv. Phys. 24 (1977) 407. 